\title{
A REMARK ON SINGULAR CALDERÓN-ZYGMUND THEORY
}

\author{
MICHAEL CHRIST AND ELIAS M. STEIN
}

ABSTRACT. It is shown that in $\mathbf{R}^{n}$ the operator

$$
H f(x)=p v \int_{-\infty}^{+\infty} f\left(x_{1}-t, \ldots x_{n}-t^{n}\right) t^{-1} d t
$$

maps $L(\log L)$ to weak $L^{1}$ locally. A slight variant of the Calderón-Zygmund procedure provides a new approach to the previously known $L^{p}$ boundedness of $H, 1<p<\infty$. Relatively sharp bounds are obtained as $p \rightarrow 1^{+}$, and extrapolation produces the result for $L(\log L)$.

Let $a=a_{1}<\cdots<a_{n} \in \mathbf{Z}$, and for all $t \in \mathbf{R}$ let $\gamma(t)=\left(t^{a_{1}}, \ldots, t^{a_{n}}\right) \in \mathbf{R}^{n}$. The $L^{p}$ boundedness of operators such as $H f(x)=p v \int f(x-\gamma(t)) t^{-1} d t$ has been studied by a number of authors $[\mathbf{S W}, \mathbf{J R d F}, \mathbf{G}, \mathbf{8}, \mathbf{C 1}, \mathbf{C 2}, \mathbf{P S}]$. In all this work there are two main steps: First one proves $L^{2}$ bounds, and then further arguments are used to pass to $L^{p}$. A prototypical technique for deducing $L^{p}$ bounds from $L^{2}$ bounds is the Calderón-Zygmund theory of singular integrals. As is well known [S1], if $K$ agrees with a function away from the origin, $\hat{K} \in L^{\infty}$ and $K$ satisfies the Hörmander condition (1) below, then convolution with $K$ is bounded on all $L^{p}$ and is of weak type on $L^{1}$. $H$ is a limiting case just outside the scope of that theory, for it is given by convolution with a distribution $K$ which is homogeneous under the family of dilations $\delta_{r} x=\left(r^{a_{1}} x_{1}, \ldots, r^{a_{n}} x_{n}\right)$, but which is equal to a difference of two Dirac measures when restricted to the unit sphere. The Hörmander condition fails, but several substitute arguments have been found [SW, JRdF, C1]. Our purpose here is to indicate a variant of the Calderón-Zygmund procedure which does apply to $H$; the main point will be that $H$ satisfies a certain generalization of the Hörmander condition. Our method applies to a more general class of convolution operators as well as to related maximal functions.

This technique appears to be slightly more precise than the reasoning used in previous studies of $H$ and related operators. Fix a bounded subset $B$ of $\mathbf{R}^{n} . L^{1, \infty}$ denotes the usual space weak $L^{1}$, equipped with the natural quasi-norm.

THEOREM 1. $H$ is a bounded operator from $L(\log L)(B)$ to $L^{1, \infty}(B)$. The same holds for the maximal function $M_{\gamma} f(x)=\sup _{r>0} r^{-1} \int_{0<t<r}|f(x-\gamma(t))| d t$.

COROLLARY. $\lim _{r \rightarrow 0} r^{-1} \int_{0<t<r} f(x-\gamma(t)) d t=f(x)$ a.e. for all $f$ locally in $L(\log L)$.

Define $|x|$ to be the reciprocal of that value of $r$ for which $\delta_{r} x \in S^{n-1}$ if $x \neq 0$, and $|0|=0$. Then a triangle inequality $|x+y| \leq C_{0}(|x|+|y|)$ holds. By a ball we

Received by the editors December 3, 1985.

1980 Mathematics Subject Classification (1985 Revision). Primary 42B20, 42B25.

Research supported in part by grants from the National Science Foundation. 
shall mean an open set $B=B(x, r)=\{y:|x-y|<r\}$, the double $B^{*}$ of $B$ is defined to be $B\left(x, 2 C_{0} r\right)$, and $B^{* *}$ is the double of $B^{*} . \varphi$ denotes a $C^{\infty}$ function supported in $B(0,1)$ satisfying $\int \varphi=1 . \varphi_{j}(x)=r^{-a} \varphi\left(\delta_{r^{-1}} x\right)$ where $r=2^{j}$ and $a=\sum a_{i}$. Let $\psi$ be $C^{\infty}$, be identically one in $B(0,1)$, and be supported in $B(0,2)$, and let $\varsigma_{j}(x)=\psi\left(\delta_{2 r} x\right)-\psi\left(\delta_{r} x\right)$ where $r=2^{-j-1}$. Finally for any tempered distribution $k$ we define $k_{j}=\zeta_{j} k$. (The symbols $k, \varphi, \zeta$ will be used in a consistent manner, so the duplication of notation need cause no confusion.) $f_{(z)}$ denotes the translate $f_{(z)}(x)=f(x+z)$, and the translate $k^{(z)}$ of a tempered distribution is defined by duality: $\left\langle f, k^{(z)}\right\rangle=\left\langle f_{(z)}, k\right\rangle$. The $L^{p}$ multiplier norm of a tempered distribution is $|k|_{p} \equiv \sup _{f \in L^{p}}\left\|f^{*} k\right\|_{p} /\|f\|_{p}$, where $\|f\|_{p}$ is the usual $L^{p}$ norm with respect to Lebesgue measure. We also write $|T|_{p}$ for the operator norm of an operator $T$, and denote the adjoint of $T$ by $T^{*}$. When $T f \equiv f^{*} k$ and $k \in L^{1}, T_{j} f$ is defined to be $f^{*} k_{j}$.

$T$ is said to satisfy the Hörmander condition if it is given by convolution with a distribution which coincides away from the origin with a function $k$ satisfying

$$
\left\|k-k^{(y)}\right\|_{L^{1}\{x:|x|>r\}}<C \text { for all }|y|<\eta r, \text { for all } r>0
$$

for some constants $C$ and $\eta$. Operators which have arisen in practice generally satisfy a slightly stronger condition:

$$
\left\|k-k^{(y)}\right\|_{L^{1}\{x:|x|>\rho r\}}<C \rho^{-\varepsilon} \text { for all }|y|<\eta r \text { and all } r>0, \rho>1
$$

for some finite positive $C, \eta, \varepsilon$. Our substitute will be

$$
\left|k_{j+i}-k_{j+i}^{(y)}\right|_{p}<C 2^{-\varepsilon i} \text { for all }|y|<\eta 2^{j} \text { and all } j \in \mathbf{Z}, i \in \mathbf{Z}^{+}
$$

for some $C, \eta, \varepsilon$. Note that when $p=1$ the $L^{1}$ and $|\cdot|_{1}$ norms coincide, and $\left(3_{p}\right)$ is essentially equivalent to (2). Many operators, including those considered in the standard Calderón-Zygmund theory, satisfy

$$
\left\|k_{j}\right\|_{L^{1}}<C .
$$

When (4) holds, $\left(3_{2}\right)$ implies $\left(3_{p}\right)$ for all $1<p<2$ by interpolation; conversely $\left(3_{p}\right)$ is equivalent to $\left(3_{q}\right)$ where $q=p^{\prime}$, and hence $\left(3_{p}\right)$ always implies $\left(3_{2}\right)$ for any $p$.

THEOREM 2. Suppose that $T f=f^{*} k$ where $k$ is a finite measure. Suppose that

$$
\left|T_{i} T_{j}^{*}\right|_{2}+\left|T_{i}^{*} T_{j}\right|_{2}<C 2^{-\varepsilon|i-j|}
$$

for all $i, j$, for some $\varepsilon>0$. Suppose that (4) and $\left(3_{2}\right)$ hold. Then $T$ is bounded on $L^{p}$ for all $1<p<\infty$, with an operator norm depending only on the constants in $\left(3_{2}\right)$, (4) and (5). Moreover, $T$ is a bounded operator from $L\left(\log L_{1}\right)(B)$ to $L^{1, \infty}(B)$ for any bounded set $B$.

For truncated Hilbert transforms $H_{a b} f(x)=\int_{a<|t|<b} f(x-\gamma(t)) t^{-1} d t$ with $\gamma$ as above, well-known bounds on Fourier transforms [SW] imply $\left(3_{2}\right)$ and (5), and therefore this theorem implies the $L^{p}$ boundedness of $H_{a b}$ uniformly in $a, b$, and hence the uniform bound $\|H f\|_{p} \leq C\|f\|_{p}$ for all $f$ in the dense subspace $C_{0}^{1}$ of $L^{p}$.

Our proof requires a parabolic Calderón-Zygmund decomposition whose proof is well known $[\mathbf{C W}]$ : 
LEMMA 1. For any $f \in L^{p}$ and $\alpha>0$ there exist $g$ and $\left\{b_{i}\right\}$ such that $f=$ $g+\sum b_{i},\|g\|_{\infty}<\alpha$, each $b_{i}$ is supported on a ball $B_{i}=B\left(x_{i}, 2^{j(i)}\right)$, the supports of the $b_{i}$ are pairwise disjoint, $\sum\left|B_{i}\right| \leq C \alpha^{-p}\|f\|_{p}^{p},\left|B_{i}\right|^{-1} \int_{B}\left|b_{i}\right|^{p} \leq C \alpha^{p}$, and no point of $\mathbf{R}^{n}$ is contained in more than $C$ of the doubles $B_{i}^{*}$.

LEMMA 2. Let $\left\{S_{j}\right\}$ be a finite set of bounded operators on a Hilbert space, and let $|T|$ denote the operator norm of an operator $T$ and $T^{*}$ its adjoint. Suppose that $\left|S_{i}^{*} S_{j}\right|+\left|S_{i} S_{j}^{*}\right| \leq C 2^{-\varepsilon|i-j|}$ for all $i, j$. Then there exists a constant $B$ depending on $C$ and on $\varepsilon$, but not on the number of $S_{j}$, such that $\left|\sum S_{j}\right| \leq C$.

Lemma 2 is due to Cotlar and Stein. At one point the classical proof of the weak-type $(1,1)$ bound, the fact that $\sum\left\|b_{i}\right\|_{L^{1}}=\left\|\sum b_{i}\right\|_{L^{1}} \leq\|f\|_{L^{1}}$ is used; unfortunately the equality fails for $L^{p}, p>1$. Instead we shall use

LEMMA 3. Suppose that operators $\left\{\Upsilon_{j}\right\}$ satisfy $\left|\Upsilon_{j}\right|_{1} \leq A_{1}$ and (5). Let $1<p \leq$ 2. Then there exists $D<\infty$ such that, for any sequence $\left\{f_{j}\right\} \subset L^{p},\left\|\sum \Upsilon_{j} f_{j}\right\|_{p} \leq$ $D\left(\sum\left\|f_{j}\right\|_{p}^{p}\right)^{1 / p}$.

PROOF. The case $p=1$ follows at once from (4) and the triangle inequality. For $p=2$, consider the operator $F=\left\{f_{j}\right\} \rightarrow \sum \Upsilon_{j} f_{j}=\Upsilon F$ from $L^{2}\left(\ell^{2}\right)$ to $L^{2}$. Set $S_{j} F=\Upsilon_{j} f_{j}$. Lemma 2, applied to the decomposition $\Upsilon=\sum S_{j}$, implies by (5) that $\Upsilon$ is bounded. Intervening values of $p$ are treated by complex interpolation; therefore $D \leq A_{1}^{1-\theta} A_{2}^{\theta}$ where $A_{2}$ is the operator norm of $\Upsilon$ from $L^{2}\left(\ell^{2}\right)$ to $L^{2}$ and $0<\theta=\theta(p) \leq 1$.

To prove Theorem 2, note that (5) implies the $L^{2}$ boundedness of $T$ by Lemma 2 . Hence by duality and the Marcinkiewicz interpolation theorem, it suffices to show that $T$ is of weak type on $L^{p}$ for each $1<p<2$. Fix such a $p$, and an $\alpha>0$. Given $f \in L^{p}$, construct $g$ and $\left\{b_{i}\right\}$ as in Lemma 1. Set $G=g+\sum\left(b_{i} * \varphi_{j(i)}\right)$; then $\|G\|_{\infty} \leq C \alpha$ since $\left\|b_{i} * \varphi_{j(i)}\right\|_{\infty} \leq C \alpha, b_{i} * \varphi_{j(i)}$ is supported on $B_{i}^{*}$ by the triangle inequality, and the $B_{i}^{*}$ have the bounded overlap property. Similarly, $\|G\|_{p} \leq C\|f\|_{p}$, so the $L^{2}$ boundedness of $T$ together with Chebychev's inequality gives $|\{x:|T G(x)|>\alpha\}| \leq C \alpha^{-p}\|f\|_{p}^{p}$. Thus it suffices to treat

$$
\begin{aligned}
\sum T b_{i}-\sum T\left(b_{i} * \varphi_{j(i)}\right) \equiv & \sum b_{i} * k *\left(\delta-\varphi_{j(i)}\right) \\
= & \sum b_{i} *\left[\sum_{s>0} k_{s+j(i)} *\left(\delta-\varphi_{j(i)}\right)\right] \\
& +\sum b_{i} *\left[\left(k \cdot \psi_{j(i)}\right) *\left(\delta-\varphi_{j(i)}\right)\right]=I+I I .
\end{aligned}
$$

$I I$ is supported on $\bigcup B_{i}^{* *}$, a set whose measure is at most $\sum\left|B_{i}^{* *}\right| \leq C \sum\left|B_{i}\right| \leq$ $C \alpha^{-p}\|f\|_{p}^{p}$. Then it suffices to show that $\|I\|_{L^{p}\left(\mathbf{R}^{n} / \cup B^{* *}\right)} \leq C\|f\|_{p}$; in fact we shall establish the same bound for $\|I\|_{L^{p}\left(\mathbf{R}^{n}\right)}$.

The smoothness hypothesis $\left(3_{2}\right)$ implies

$$
\left|k_{j+s} *\left(\delta-\varphi_{j}\right)\right|_{2} \leq C 2^{-\varepsilon s} \text { for all } j \in \mathbf{Z}, s \in \mathbf{Z}^{+} .
$$

Let $a_{j}=\sum_{j(i)=j} b_{i}$. Then $I=\sum_{s>0}\left[\sum_{j} a_{j} * k_{j+s} *\left(\delta-\varphi_{j}\right)\right]$. Fix $s$ and set $\Upsilon_{j} f=f * k_{j+s} *\left(\delta-\varphi_{j}\right)$. (5), (6) and Lemma 2 imply that the operator norm of $\Upsilon$ from $L^{2}\left(\ell^{2}\right)$ to $L^{2}$ is $\leq C 2^{-\sigma s}$ for some $\sigma>0(\sigma<\varepsilon / 2)$. Therefore, $\left\|\sum \Upsilon_{j} a_{j}\right\|_{p} \leq$ $C 2^{-\theta \sigma s}\left(\sum\left\|a_{j}\right\|_{p}^{p}\right)^{1 / p} \leq C 2^{-\theta \sigma s}\|f\|_{p}$ for some $0<\theta<1$, and summing over $s$ concludes the proof. 
The result for $L(\log L)$ is proved by extrapolating the bounds obtained from the above argument. Suppose that $f \in L(\log L)(B)$ and $\alpha>0$ are given. Apply Lemma 1 with $p=1$. Then the measure of the set on which $|T G|$ is greater than $\alpha / 2$ is at most $C \alpha^{-1}\|f\|_{L^{1}} \leq C \alpha^{-1}\|f\|_{L(\log L)}$. Let $E$ be the union of the $B_{i}^{* *}$. Then $|E| \leq C \alpha^{-1}\|f\|_{L^{1}} \leq C \alpha^{-1}\|f\|_{L(\log L)(B)}$ since $B$ is bounded. Hence it suffices to show

$$
\|I\|_{L^{1}(B)} \leq C\left\|\sum b_{i}\right\|_{L(\log L)}
$$

with a bound $C$ independent of $\alpha$ and of the balls $B_{i}$. For all $p,\left\|\sum b_{i}\right\|_{L^{p}} \leq C\|f\|_{L^{p}}$ with $C$ independent of $p$. The above argument gives $\|I\|_{L^{p}} \leq C(p-1)^{-1}\left\|\sum b_{i}\right\|_{L^{p}}$ with $C$ independent of $p$ for all $1<p \leq 2$. Consider the linear operator $\left\{b_{i}\right\} \rightarrow I$ from $L^{p}\left(\bigcup B_{i}\right)$ to $L^{p}\left(\mathbf{R}^{n}\right)$. By the extrapolation theorem of Yano [Y] it is bounded from $L(\log L)\left(\bigcup B_{i}\right)$ to $L^{1}(B)$.

The maximal function $M_{\gamma}$ is treated by introducing a square function and applying the same reasoning to it. Fix a nonnegative $C^{\infty}$ auxiliary function $\zeta$ supported on $\left(\frac{1}{4}, 2\right)$ and positive on $\left[\frac{1}{2}, 1\right]$. Define measures $\mu_{j}$ on $\mathbf{R}^{n}$ by $\left\langle f, \mu_{j}\right\rangle=$ $\int f(\gamma(t)) \varsigma\left(2^{-j} t\right) 2^{-j} d t$. Then for all nonnegative $f$ there is a pointwise bound $M_{\gamma} f(x) \leq C \sup _{j}\left|f * \mu_{j}(x)\right|$. To bound the latter maximal function we proceed as for $H$. Since it is certainly bounded on $L^{\infty}$ matters reduce to the estimation of the analogue of $I$, the sum over all integers $s>0$ of the square functions $S_{s}(x)=\left(\sum_{m \in \mathbf{Z}}\left|\sum_{i: j(i)=m} b_{i} *\left(\delta-\varphi_{j(i)}\right) * \mu_{j(i)+s}(x)\right|^{2}\right)^{1 / 2}$ (for more details see [C4]). The $L^{2}$ bound $\left\|S_{s}\right\|_{L^{2}}^{2} \leq C 2^{-\varepsilon s} \sum\left\|b_{i}\right\|_{L^{2}}^{2}$ follows as before. The easy $L^{1}$ bound $\left\|S_{s}\right\|_{L^{1}} \leq C \sum\left\|b_{i}\right\|_{L^{1}} \leq C\|f\|_{L^{1}}$ is obtained by dominating the $\ell^{2}$ norm in the definition of $S_{s}$ by the $\ell^{1}$ norm and applying the triangle inequality.

Analogues of the proposition hold in greater generality, for instance on homogeneous nilpotent Lie groups. Thus we have another proof of the $L^{p}$ results of $[\mathbf{C 1}$, and in fact an extension of the differentiation results of [C1 and C3] to $L(\log L)$ since the appropriate analogues of the $L^{2}$ hypotheses $\left(3_{2}\right)$ and $(5)$ are verified there. However, the method does not apply to those curves treated in [8], which may contain line segments and hence lack curvature. Moreover, hypothesis (4) fails to hold for certain more singular variants considered in $[\mathbf{S W}]$ implicitly and $[\mathbf{G}]$ explicitly, where even away from the origin $k$ is a distribution of positive order.

It is not known whether operators of the types considered here are of weak type $(1,1)$. A weaker conjecture would be that they map $L(\log L)(B)$ to $L^{1}(B)$, rather than merely to weak $L^{1}$.

In dimension $n=2$ results more refined than those in this note have already been obtained, for the maximal function $M_{\gamma}$, in [C4].

\section{REFERENCES}

[8] H. Carlsson, M. Christ, A. Córdoba, J. Duoandikoetxea, J. L. Rubio de Francia, J. Vance, S. Wainger, and D. Weinberg, $L^{p}$ estimates for maximal functions and Hilbert transforms along flat convex curves in $\mathbf{R}^{2}$, Bull. Amer. Math. Soc. 14 (1986), 263-267.

[C1] M. Christ, Hilbert transforms along curves. I: Nilpotent groups, Ann. of Math. (2) 122 (1985), 575-596.

[C2] _ _ Hilbert transforms along curves. II: A flat case, Duke Math. J. 52 (1985), 887-894.

[C3] _ Differentiation along variable curves and related singular integral operators, announcement.

[C4] _ Weak type $(1,1)$ bounds for rough operators, preprint. 
[CW] R. R. Coifman and G. Weiss, Analyse noncommutative sur certains espaces homogenes, Lecture Notes in Math., vol. 242, Springer-Verlag, Berlin and New York, 1971.

[G] A. Greenleaf, Singular integral operators with conical singularities, preprint.

[JRdF] J. Duoandikoetxea and J. L. Rubio de Francia, Maximal and singular integral operators via Fourier transform estimates, Invent. Math. 84 (1986), 541-561.

[PS] D. H. Phong and E. M. Stein, Hilbert integrals, singular integrals, and Radon transforms, Acta Math. (to appear).

[S1] E. M. Stein, Singular integrals and differentiability properties of functions, Princeton Univ. Press, Princeton, N.J., 1970.

[SW] E. M. Stein and S. Wainger, Problems in harmonic analysis related to curvature, Bull. Amer. Math. Soc. 84 (1978), 1239-1295.

[Y] S. Yano, An extrapolation theorem, J. Math. Soc. Japan 3 (1951), 296-305.

Department of Mathematics, Princeton University, Princeton, New Jersey 08540 (Current address of E. M. Stein)

Current address (Michael Christ): Department of Mathematics, University of California, Los Angeles, California 90024 